\title{
Original $\mid$ PHASE II TRIAL OF CISPLATIN AND VINORELBINE AS FIRST-LINE Article THERAPY IN MALIGNANT PLEURAL MESOTHELIOMA
}

\author{
Salah El-Mesidy ${ }^{1}$, Amr El-Kashef ${ }^{1}$, Amr Sakr ${ }^{1}$, Mohamed Abd El-Hakim²
}

${ }^{1}$ Kasr El-Aini Center of Clinical Oncology, Faculty of Medicine, ${ }^{2}$ Chest Department, Faculty of Medicine, Cairo University, Egypt

\begin{abstract}
Aim of the Work: The aim of the current study is to focus on treatment response in patients with malignant pleural mesothelioma (MPM) treated with combination chemotherapy using cisplatin plus vinorelbine. Secondary endpoints included, toxicity, progression-free and overall survival.

Patients and Methods: This prospective study included 26 patients with histologically proven unresectable MPM treated at Kasr El-Aini Center of Clinical Oncology and Nuclear Medicine (NEMROCK) from March 2003 to August 2004. Patients were assigned to receive cisplatin $75 \mathrm{mg} / \mathrm{m}^{2}$ on day one and vinorelbine $25 \mathrm{mg} / \mathrm{m}^{2}$ on days one and 8 every three weeks.
\end{abstract}

Results: All 26 patients had measurable disease and were assessed for response. Six patients had partial response $(23 \%), 14$ patients had stable disease $(54 \%)$, and six patients had disease progression on therapy (23\%). Toxicity was acceptable and no treatment-related deaths occurred. The median progression-free survival was 5.15 months and the median overall survival for was 10.3 months, with a $42.3 \%$ one-year survival.

Conclusion: Cisplatin-vinorelbine combination is an effectve regimen for management of malignant pleural mesothelioma with a tolerable toxicity profile. Further studies with a larger number of patients is necessary.

Key Words: Mesothelioma, cisplatin, vinorelbine.

Corresponding Author: Amr El-Kashef, kasr al-Aini: Hospital, Cairo, Egypt, Tel.:0101450259,

E-mail: amrsakr@yahoo.com

\section{INTRODUCTION}

Malignant pleural mesothelioma (MPM) is an aggressive tumor of the pleura and peritoneum. It is uncommon but is increasing in incidence, particularly in regions where occupational exposure to asbestos has been prominent $t^{1,2}$. It is generally considered to be resistant to therapy and radical surgery is not possible for most patients. Palliative radiotherapy may provide pain relief but does not prolong survival. ${ }^{3}$

The natural history is characterized by a median survival of 9-14 months, with $<5 \%$ five years survivors. Objective response rates of $16-48 \%$ and median survivals of 9.4-11.2 months have been reported with the combination of cisplatin (CDDP) and gemcitabine (GEM) in malignant mesothelioma. ${ }^{4,5}$

Vinorelbine is a semi-synthetic derivative of vinblastine which is structurally modified in the catharanthine nucleus. Recently, an objective response rate of $24 \%$ has been reported with the single agent vinorelbine in a single institution study ${ }^{6}$. In Egypt, the combination of cisplatin plus vinorelbine was studied in a relatively small number of patients (16 patients) with promising results ${ }^{7}$. Therefore, combination chemotherapy using cisplatin and vinorelbine seems to have a potential anti-tumor activity against MPM.

The primary endpoint of the current prospective study is to focus on treatment response in patients with MPM treated at Kasr El-Aini Center of Radiation Oncology and Nuclear Medicine (NEMROCK) with combination chemotherapy using cisplatin plus vinorelbine. Secondary endpoints included toxicity, progression-free and overall survival.

\section{PATIENTS AND METHODS}

The current prospective study included 26 patients with histologically proven unresectable MPM treated at NEMROCK from March 2003 to August 2004. The study has appropriate institutional ethical review board approval, and all patients provided their written, informed consent. The inclusion crieteria included: Age 70 years old, performance status (PS) of 0-2 according to The Eastern Cooperative Oncology Group (ECOG) scale, histologically confirmed diagnosis of unresectable MPM. adequate organ functions i.e. adequate bone marrow function [defined as total leukocyte count $\geq 4 \times 10^{9} / 1$, 
absolute neutrophil count (ANC) $\geq 2 \times 10^{9} / 1$, platelet count $100 \times 109 / 1$ and hemoglobin level $9.5 \mathrm{~g} / \mathrm{dl}$ ]; adequate renal function (defined as serum creatinine level upper normal limit for the laboratory, or creatinine clearance $60 \mathrm{ml} / \mathrm{min}$ ), adequate hepatic function (defined as total bilirubin level 1.5 times the upper limit of normal and serum AST and/or ALT and alkaline phosphatase levels two times the upper normal limit for the laboratory). no associated comorbidities e.g. cardiac or hepatic diseases. No prior chemotherapy or radiotherapy. The clinical or pathological stage of the disease was based on the International Mesothelioma Interest Group (IMIG) staging system. ${ }^{8}$

\section{Treatment schedule:}

Eligible patients received the following regimen, cisplatin $75 \mathrm{mg} / \mathrm{m}^{2}$ on day one and vinorelbine $25 \mathrm{mg} / \mathrm{m}^{2}$ on days one and 8 every three weeks. The blood counts and chemistries were examined before day one and day 8 of each cycle. If grade three or more leukopenia and neutropenia, grade two or more thrombocytopenia, or grade two or more non-hematological toxicities occurred on day 8 , the treatment on that day is to be skipped. Prophylactic granulocyte colony-stimulating factor (G$\mathrm{CSF}$ ) during any cycle is not be used routinely, may be used only for patients with ANC $<0.5$ x 109/1, neutropenic fever or documented infection while neutropenic. In the absence of either disease progression or unacceptable toxicity levels, patients were scheduled to receive the treatment for six cycles.

\section{Tumor Assessment During And After Treatment:}

Patients were assessed for response by CT chest scan after three cycles of chemotherapy and at the end of treatment. The new guidelines were used to evaluate the response to treatment in solid tumors (Response Evaluation Criteria in Solid Tumors) ${ }^{9}$. Spiral CT scans were used to measure the response. If there were clearly identifiable mass lesions, then all such lesions up to a maximum of five were selected for measurement of their longest diameter. If there was no separately identifiable mass lesions, then the thickness of circumferential pleural tumor was measured at three separate levels on transverse sections, The sum of these measurements was taken as the baseline sum longest diameter. Repeated measurements were made at the same levels on subsequent scans.

The baseline sum longest diameter was used to characterize response as follows: One complete response was defined as the disappearance of all target lesions, two partial response was defined as a decrease of at least $30 \%$ in the sum longest diameter. Three progressive disease was defined as an increase of at least $20 \%$ in the sum longest diameter (taking as a reference the smallest sum longest diameter recorded before starting treatment), or the appearance of one or more new lesions. Four patients were considered to have stable disease if neither sufficient shrinkage to qualify for partial response nor sufficient increase to qualify for progressive disease had occurred, (taking as a reference the smallest sum longest diameter recorded before starting treatment). Treatment toxicity was evaluated according to the National Cancer Institute, Common Toxicity Criteria version 2.0 grading system. ${ }^{10}$

After completion of the study treatment, patients were followed up every three months with chest CT scans for one year. Patients were also observed for survival until death or last contact if still alive. Second-line therapy was not planned in this trial. Radiotherapy was given as a part of palliative treatment for patients with progressive disease and uncontrollable symptoms.

\section{Statistical considerations}

Major endpoints of the study were, response rate, overall survival, and progression free survival. The Kaplan-Meier method was used for calculation of overall survival and progression free survival. Time to tumor progression was estimated from the date of first treatment to first evidence of disease progression. Survival was estimated from the date of first treatment to death or last follow-up visit. ${ }^{11}$

\section{RESULTS}

The current prospective study included 26 eligible patients. The median age of the patients was 40 years (range 18 - 66 years). Sixteen cases were males (61.5\%) and 10 cases were females $(38.5 \%)$. Twenty patients lived in Helwan and four patients in Shoubra Al-Khima (areas which are well-known for a high pollution rate and occupational exposure to carcinogens linked with the risk of development of mesothelioma).

Histologic subtypes were distributed as follows; epithelioid in 17 patients $(65.4 \%)$, sarcomatoid in six patients $(23.1 \%)$ and biphasic in three patients $(11.5 \%)$. The majority of patients (16 patients) had performance status of two according to ECOG scale. According to the International Mesothelioma Interest Group (IMIG) staging system, 8 patients $(30.8 \%)$ had stage II disease and 10 patients $(38.5 \%)$ had stage III disease. The characteristics of the treated patients are listed in table 1.

Twenty-six eligible patients were assessed for radiologic response. Of those, there were 6 partial responses (23\%) Figure 1 and 2 show sequential spiral CT chest scans of one of the patients who achieved partial response, 14 patients (54\%) had stable disease, and six (23\%) experienced disease progression on treatment. Respo were observed in all three histologic subtypes of mesothelioma. 
Table 1: Patient characteristics.

\begin{tabular}{lcc}
\hline Characteristic & $\begin{array}{c}\text { No. of patients } \\
(\mathbf{N}=\mathbf{2 6})\end{array}$ & $\%$ \\
\hline Age & 40 & \\
$\quad$ Median & $18-66$ & \\
Range & & \\
Sex & 16 & 61.5 \\
Male & 10 & 23.0 \\
Female & & \\
Histologic type & 17 & 65.4 \\
Epithelioid & 6 & 23.0 \\
sarcomatoil & 3 & 11.6 \\
Biphasic & & \\
Performance status & 2 & 7.7 \\
0 & 8 & 30.8 \\
1 & 16 & 61.5 \\
2 & & \\
IMG stage & 2 & 3.7 \\
Ib & 8 & 38.5 \\
II & 10 & 23.0 \\
III & 6 & \\
IV & & \\
\hline & & \\
& &
\end{tabular}

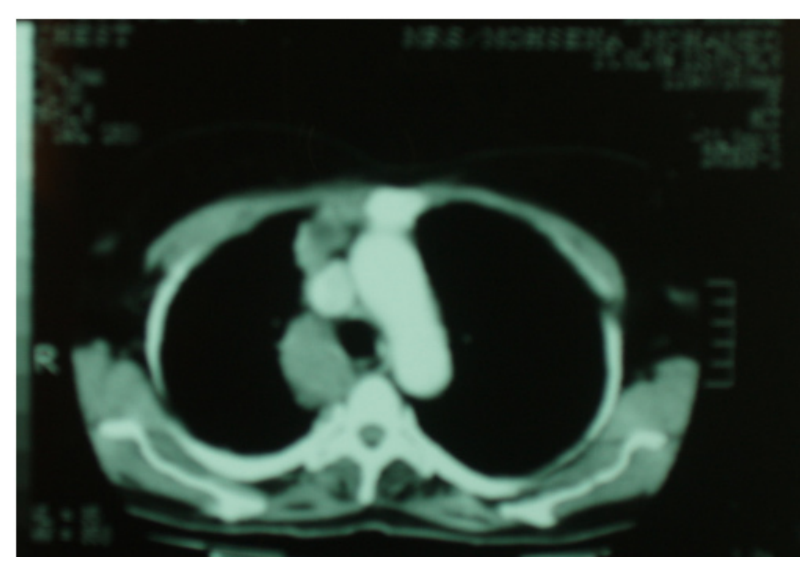

Fig. 1: CT Chest of one patient before treatment.

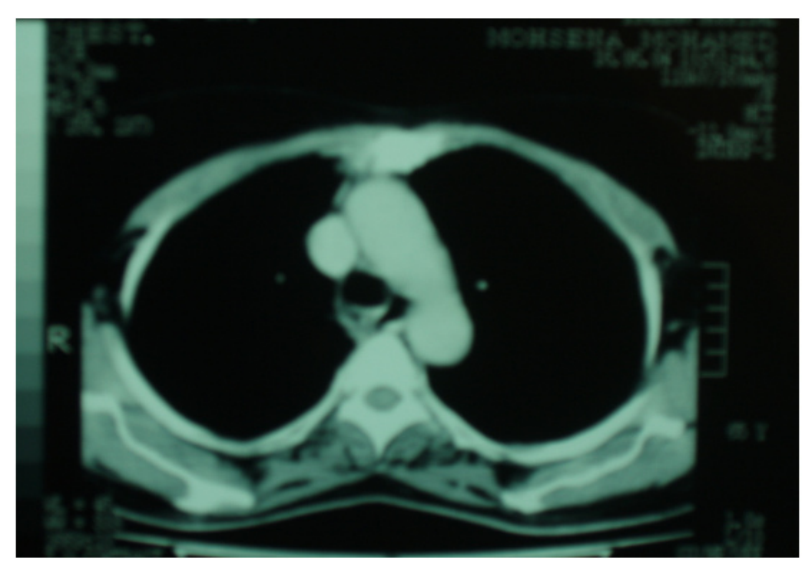

Fig. 2: CT Chest of the same patient after three cycles of chemotherapy.
Specific treatment - related toxicities are listed in table2, in general the regimen was safe and no major toxicity that necessitated discontinuation of cheomotherapy protocol occurred. Grade three neutropenia was seen in three patients $(11.5 \%)$, only one case developed grade four toxicity and granulocyte colony stimulating factor (G-CSF) was administered. Grade three anemia and thrombocytopenia were detected in two $(7.7 \%)$ and one $(3.8 \%)$ patient respectively. Grade 2 and three nephrotoxicity was observed in three patients (11.5\%). Only one patient had hypersensitivity reaction, whereas, 7 patients $(26.3 \%)$ developed local reaction at the site of injection .

Table 2: Treatment related toxicities.

\begin{tabular}{|c|c|c|c|c|}
\hline Toxicity & Grade 1 & Grade 2 & Grade 3 & Grade 4 \\
\hline Anemia & $\begin{array}{c}9 \\
34.6 \%\end{array}$ & $\begin{array}{c}10 \\
38.46 \%\end{array}$ & $\begin{array}{c}2 \\
7.6 \%\end{array}$ & \\
\hline Neutropenia & $\begin{array}{c}6 \\
23 \%\end{array}$ & $\begin{array}{c}4 \\
15.38 \%\end{array}$ & $\begin{array}{c}3 \\
11.5 \%\end{array}$ & $\begin{array}{c}1 \\
3.8 \%\end{array}$ \\
\hline Thrombocytopenia & $\begin{array}{c}3 \\
11.5 \%\end{array}$ & $\begin{array}{c}2 \\
7.6 \%\end{array}$ & $\begin{array}{c}1 \\
3.8 \%\end{array}$ & \\
\hline Nausea/Vomiting & $\begin{array}{c}10 \\
38.46 \%\end{array}$ & $\begin{array}{c}4 \\
15.38 \%\end{array}$ & $\begin{array}{c}2 \\
7.6 \%\end{array}$ & \\
\hline Diarrhea & $\begin{array}{c}3 \\
11.5 \%\end{array}$ & $\begin{array}{c}1 \\
3.8 \%\end{array}$ & & \\
\hline Mucositis & $\begin{array}{c}1 \\
3.8 \%\end{array}$ & & & \\
\hline Neurotoxicity & $\begin{array}{c}5 \\
19.23 \%\end{array}$ & $\begin{array}{c}4 \\
15.38 \%\end{array}$ & $\begin{array}{c}2 \\
7.6 \%\end{array}$ & \\
\hline Asthenia & $\begin{array}{c}5 \\
19.23 \%\end{array}$ & $\begin{array}{c}5 \\
19.23 \%\end{array}$ & $\begin{array}{c}3 \\
11.5 \%\end{array}$ & \\
\hline $\begin{array}{l}\text { Hypersensitivity } \\
\text { reaction }\end{array}$ & $\begin{array}{c}1 \\
3.8 \%\end{array}$ & & & \\
\hline Nephrotoxicity & & $\begin{array}{c}2 \\
7.6 \%\end{array}$ & $\begin{array}{c}1 \\
3.8 \%\end{array}$ & \\
\hline Ototoxicity & & $\begin{array}{c}1 \\
3.8 \%\end{array}$ & $\begin{array}{c}1 \\
3.8 \%\end{array}$ & \\
\hline $\begin{array}{l}\text { Injection site } \\
\text { reaction }\end{array}$ & $\begin{array}{c}2 \\
7.6 \%\end{array}$ & $\begin{array}{c}5 \\
19.23 \%\end{array}$ & & \\
\hline
\end{tabular}

The median survival (from the date of first treatment to the date death or last follow-up visit) was 10.3 months (Figure 3) and the survival rate at one year was $42.3 \%$. The median progression - free survival (from the date of first treatment to the date of first evidence of disease progression) was 5.15 months (Figure 4) 


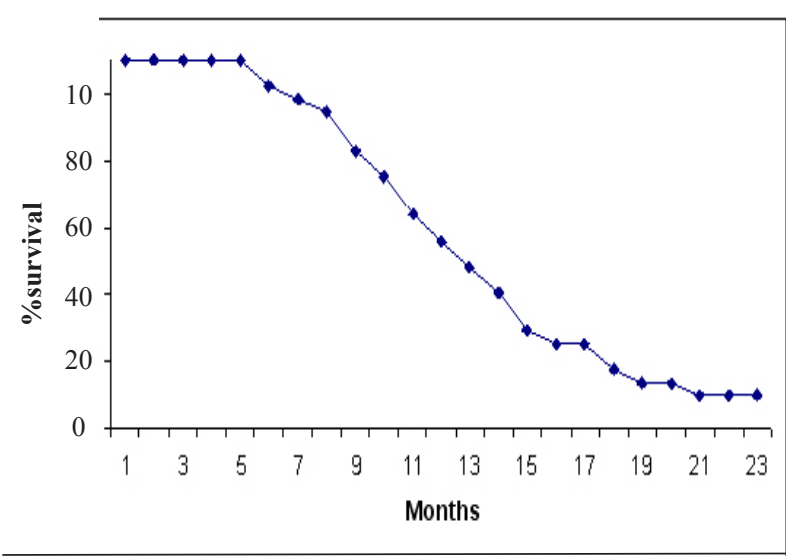

Fig. 3: Overall survival.

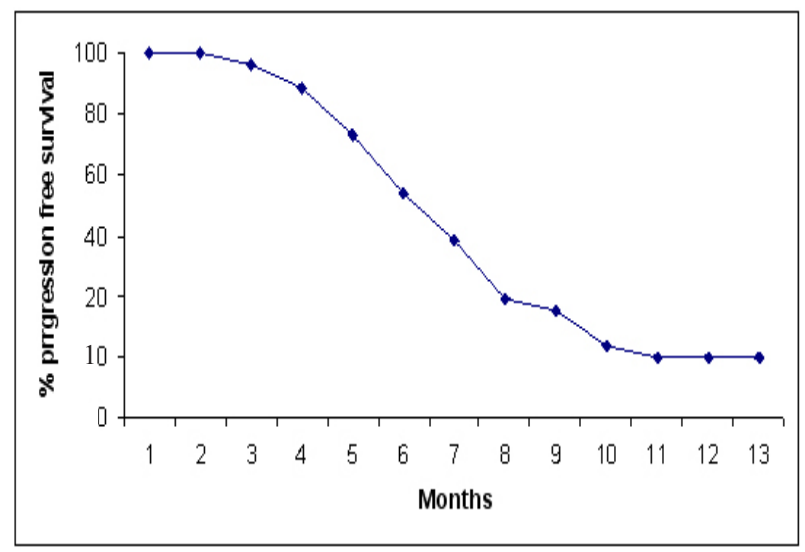

Fig. 4: Progression free survival.

\section{DISCUSSION}

The current prospective study included 26 patients with histologically proven unresectable MPM treated at NEMROCK with combination chemotherapy using cisplatin plus vinorelbine. The median age of the patients was 40 years (range $18-66$ years) which is not comparable to that of the study done by Maruyama et al. ${ }^{2}$, Steele et al. ${ }^{6}$, and Ceresoli et al. ${ }^{12}$, where the median age at diagnosis was 53 years (range $34-67$ ), 58 years (range $29-77$ ), and 65 years (range 38 -79), respectively. This may be explained by the tendency of mesthelioma to occur at younger age in our series (20-40 years) due to earlier and prolonged exposure to carcinogens, mainly asbestos. Twenty patients were living in Helwan and four patients in Shoubra Al-Khima, which are well known areas for a high pollution rate and occupational exposure to carcinogens.

The distribution of the histologic subtypes was, epithelioid in 17 patients $(65.4 \%)$, sarcomatoid in 6 patients $(23.1 \%)$ and biphasic in three patients $(11.5 \%)$. This coincides with the work of Maruyama et al. ${ }^{2}$, epithelioid type in 58\%, and Steele et al. ${ }^{6}$, epithelioid type in $59 \%$, but differs from the study done by Ceresoli et al. ${ }^{12}$, epithelioid type in $78 \%$ and sarcomatoid type in only $7 \%$, which may have a reflection on better treatment results. Furthermore, in the current study, 8 patients $(30.8 \%)$ had stage II disease and 10 patients $(38.5 \%)$ had stage III disease which is more or less comparable to the findings in the previously mentioned studies.

The response rate of the combination cisplatin plus vinorelbine was $23 \%$ partial response, $54 \%$ stable disease and $23 \%$ of patients experienced disease progression on treatment . Responses were observed in all three histologic subtypes of mesothelioma. The median survival was 10.3 months and the survival rate at one year was $42.3 \%$. The median progression-free survival was 5.15 months. Other studies had reported mixed results both in single agent studies as well as in combination chemotherapy studies.

In single-agent chemotherapy studies, many cytotoxic agents were used in phase II studies. Vogelzang et al. ${ }^{13}$ used high-dose paclitaxel with granulocyte colonystimulating factor in 35 patients with mesothelioma and reported a response rate of $9 \%$ in assessable patients. O'Reilly et al. ${ }^{14}$ used carboplatin with interferon alfa-2a in patients with advanced mesothelioma and showed a response rate of $7 \%$ equivalent to that seen with singleagent carboplatin. The antimetabolite gemcitabine was evaluated by several groups. Van Meerbeeck et al. ${ }^{15}$ tested gemcitabine $1,250 \mathrm{mg} / \mathrm{m}^{2}$ on days 1,8 , and 15 of a 28-day cycle in 27 patients with MPM, with an objective response rate of $7 \%$ and a median survival of 8 months. Steele et al. ${ }^{6}$ evaluated the response rate of single agent vinorelbine given as cycles of $30 \mathrm{mg} / \mathrm{m}^{2}$ weekly for 6 weeks to 29 patients with MPM. Partial response was evident in $24 \%$ of patients, stable disease in $55 \%$ and disease progression in $21 \%$, which is comparable with the response rate reported in our study. Pemetrexed, a novel multitargeted antifolate, was shown to have activity as a single agent in a phase II trial in patients with MPM in the study coducted by Adjei ${ }^{16}$. It was also evaluated by Scagliotti et al. ${ }^{17}$ in 64 patients with $14.1 \%$ response rate and median overall survival of 10.7 months.

Combination chemotherapy can produce response rates greater than $20 \%$, although toxicity accumulates more often. Kasseyet et al. ${ }^{18}$ reported a $38 \%$ response rate and a median survival time of 16 months in 45 patients treated with cisplatin, etoposide. Byrne et al. ${ }^{19}$ used gemcitabine $1,000 \mathrm{mg} / \mathrm{m}^{2}$ on days 1,8 , and 15 of a 28-day cycle in combination with cisplatin $100 \mathrm{mg} / \mathrm{m}^{2}$ to give a response rate of $47.6 \%$, although hematologic toxicity was appreciable. The median survival from start of treatment was 41 weeks. Other trials using gemcitabine and cisplatin regimen did not achieve such a response, in the trial done by Van Haarst et al. ${ }^{5}$, only a $16 \%$ response rate was reported.

In the Egyptian trial conducted by Elsharawy et al. ${ }^{7}$, the combination of cisplatin plus vinorelbine was studied in a relatively small number of patients (16 patients) with promising results (18.75\% partial response, $18.75 \%$ 
minimal response which is less than $50 \%$ reduction and $43.75 \%$ stable disease), which is comparable to the results obtained in our study. Fennell et al. ${ }^{1}$ tested the combination of vinorelbine and oxaliplatin in 26 patients with MPM, there were 6 partial responses, 17 patients with stable disease, and three patients with progressive disease. Response rate was $23 \%$, progression-free survival was 4.7 months, overall survival was 8.8 months, and the survival rate at one year was $27 \%$.

Furthermore, triplet chemotherapy with vinorelbine, cisplatin, and gemcitabine was used for the treatment of MPM .The overall response rate was $58 \%$, the median survival time and survival rate at two years were 11 months and $50 \%$, respectively ${ }^{2}$. Recently, the combination of pemetrexed and carboplatin was studied in 102 patients, two patients experienced a complete response, whereas a partial response was achieved in 17 patients, 48 patients had stable disease. Median progression-free survival was 6.5 months, and median overall survival was 8.8 months. ${ }^{12}$

The combination of cisplatin plus vinorelbine was generally safe and well tolerated in the majority of patients. The toxicity was mainly hematologic, grade three neutropenia occurred in three patients $(11.5 \%)$ and only one case developed grade four toxicity. Grade three anemia was detected in two patients (7.7\%). Nonhematologic toxicity was mild, nephrotoxicity grade three was only documented in one patient and three patients developed phlebitis (it is recommended to give vinorelbine through a central line whenever possible).

This toxicity profile is more or less comparable to that of similar studies. Fennell et al. ${ }^{1}$ reported $\mathrm{G} 3$ and 4 neutropenia $18 \%$, phlebitis $12 \%$, malaise $12 \%$ anorexia $12 \%$, nausea and vomiting $12 \%$, and constipation $6 \%$ of the total cycles ${ }^{1}$. Toxicity was mild also in the study conducted by Ceresoli et al. ${ }^{12}$, with grade three or four neutropenia occurring in $9.7 \%$ of total cycles and grade three or four anemia occurring in $3.5 \%$ of total cycles. Nonhematologic toxicity was negligible. However, with the use of triplet chemotherapy (vinorelbine, cisplatin, and gemcitabine), toxicity was more but manageable. No toxic deaths occurred. Grade 3 and 4 leukopenia, neutropenia, anemia and thrombocytopenia occurred in $50,92,33$ and $17 \%$, respectively, 8 patients received GCSF out of 12 patients during 10 cycles out of 35 . The median duration of grade four neutropenia was three days (range: 2-7 days). Severe non-hematological toxicity was uncommon. ${ }^{2}$

\section{CONCLUSION}

Cisplatin-vinorelbine combination chemotherapy is an effectve regimen for management of MPM with a tolerable toxicity profile. Further studies with a larger number of patients is necessary.
Although the new chemotherapy regimens clearly represent a step forward in the treatment of MPM, the prognosis for these patients remains poor and new therapeutic strategies are eagerly awaited.

\section{REFERENCES}

1. Fennell DA, C-Steele JP, Shamash J, Sheaff MT, Evans MT, Goonewardene TI, et al. Phase II trial of vinorelbine and oxaliplatin as first-line therapy in malignant pleural mesothelioma. Lung Cancer 2005 Feb;47(2):277-81.

2. Maruyama R, Shoji F, Okamoto T, Miyamoto T, Miyake T, Nakamura T, et al. Triplet chemotherapy with cisplatin, gemcitabine and vinorelbine for malignant pleural mesothelioma. Jpn J Clin Oncol 2005 Aug;35(8):433-8.

3. Bissett D, Macbeth FR, Cram I. The role of palliative radiotherapy in malignant mesothelioma. Clin Oncol (R Coll Radiol) 1991 Nov;3(6):315-7.

4. Nowak AK, Byrne MJ, Williamson R, Ryan G, Segal A, Fielding $\mathrm{D}$, et al. A multicentre phase II study of cisplatin and gemcitabine for malignant mesothelioma. Br J Cancer 2002 Aug 27;87(5):4916.

5. Van Haarst JM, Baas P, Manegold Ch, Schouwink JH, Burgers JA, de Bruin HG, et al. Multicentre phase II study of gemcitabine and cisplatin in malignant pleural mesothelioma. Br $\mathrm{J}$ Cancer 2002 Feb 1;86(3):342-5.

6. Steele JP, Shamash J, Evans MT, Gower NH, Tischkowitz MD, Rudd RM. Phase II study of vinorelbine in patients with malignant pleural mesothelioma. J Clin Oncol 2000 Dec 1;18(23):3912-7.

7. El Shaarawi EA, Fouad IM, Shaaban M. A phase II study of the combination of cisplatin plus vinorelbine in the treatment of unresectable pleural malignant mesothelioma. Egypt J Chest Dis and Tuberc. 2000;49(2):137-43.

8. Rusch VW. A proposed new international TNM staging system for malignant pleural mesothelioma from the International Mesothelioma Interest Group. Lung Cancer 1996 Feb;14(1):112.

9. Therasse P, Arbuck SG, Eisenhauer EA, Wanders J, Kaplan RS, Rubinstein L, et al. New guidelines to evaluate the response to treatment in solid tumors. European Organization for Research and Treatment of Cancer, National Cancer Institute of the United States, National Cancer Institute of Canada. J Natl Cancer Inst 2000 Feb 2;92(3):205-16.

10. Cancer Therapy Evaluation Program. Common toxicity criteria manual: Common toxicity criteria, version 2.0. 1999.

11. Miller AB, Hoogstraten B, Staquet M, Winkler A. Reporting results of cancer treatment. Cancer 1981 Jan 1;47(1):207-14. 
12. Ceresoli GL, Zucali PA, Favaretto AG, Grossi F, Bidoli P, Del Conte G, et al. Phase II study of pemetrexed plus carboplatin in malignant pleural mesothelioma. J Clin Oncol 2006 Mar 20;24(9):1443-8.

13. Vogelzang NJ, Herndon JE, $2^{\text {nd }}$, Miller A, Strauss G, Clamon G, Stewart FM, et al. High-dose paclitaxel plus G-CSF for malignant mesothelioma: CALGB phase II study 9234. Ann Oncol 1999 May;10(5):597-600.

14. O'Reilly EM, Ilson DH, Saltz LB, Heelan R, Martin L, Kelsen DP. A phase II trial of interferon alpha-2a and carboplatin in patients with advanced malignant mesothelioma. Cancer Invest 1999;17(3):195-200.

15. van Meerbeeck JP, Baas P, Debruyne C, Groen HJ, Manegold C, Ardizzoni A, et al. A Phase II study of gemcitabine in patients with malignant pleural mesothelioma. European Organization for Research and Treatment of Cancer Lung Cancer Cooperative Group. Cancer 1999 Jun 15;85(12):2577-82.
16. Adjei AA. Pemetrexed: A multitargeted antifolate agent with promising activity in solid tumors. Ann Oncol 2000 Oct;11(10):1335-41.

17. Scagliotti GV, Shin DM, Kindler HL, Vasconcelles MJ, Keppler U, Manegold C, et al. Phase II study of pemetrexed with and without folic acid and vitamin B12 as front-line therapy in malignant pleural mesothelioma. J Clin Oncol 2003 Apr 15;21(8):1556-61.

18. Kasseyet S, Astoul P, Boutin C. Results of a phase II trial of combined chemotherapy for patients with diffuse malignant mesothelioma of the pleura. Cancer 1999 Apr 15;85(8):1740-9.

19. Byrne MJ, Davidson JA, Musk AW, Dewar J, van Hazel G, Buck $\mathrm{M}$, et al. Cisplatin and gemcitabine treatment for malignant mesothelioma: A phase II study. J Clin Oncol 1999 Jan;17(1):2530. 